\title{
La nación bajo examen. La historiografía sobre el nacionalismo y la identidad nacional en el siglo XIX chileno
}

\author{
Gabriel Cid
}

Programa de Historia de las Ideas Políticas en Chile, Universidad Diego Portales, Santiago, Chile. Email: gabriel.cid@udp.cl

Resumen: El propósito de este ensayo es analizar de forma panorámica la producción historiográfica relativa a los fenómenos de la construcción de nación, el nacionalismo y la identidad nacional en Chile durante el siglo XIX. El artículo se propone establecer el "estado de la cuestión” en estos problemas, poniendo especial énfasis en los debates centrales de la historiografía, los aportes más destacados, las limitaciones teóricas y las posibilidades metodológicas y temáticas que ofrece la disciplina histórica para abordar este problema.

Palabras clave: Construcción de nación, Nacionalismo, Identidad Nacional, Historiografía, Chile.

\section{The nation under examination: the historiography on nationalism and national identity in the Chilean nineteenth-century}

Abstract: The purpose of this essay is to examine the evolution of the historiography concerning the matters of nation-building, nationalism and the Chilean national identity during the nineteenth century. This article aims to identify the state of the matter according with these problems, focuses on the major historiographical discussions, principal contributions, theoretical limits and methodological possibilities that historiography offers to analyze these main issues. Chile.

Key words: Nation-building, nationalism, national identity, historiography,

\section{A nação em análise: a historiografia sobre o nacionalismo e identidade nacional no Chile do século XIX}

Resumo: O objetivo deste ensaio é examinar a evolução da historiografia sobre as questões de construção da nação, nacionalismo e identidade nacional chilena durante o século XIX. Este artigo tem como objetivo identificar o estado da matéria de acordo com estes problemas, centra-se nas grandes discussões historiográficas, as contribuições principais, limites teóricos e possibilidades metodológicas que a historiografia oferece para analisar estas questões principais. 
Palavras-chave: construção de nação, Nacionalismo, Identidade Nacional Historiografia, Chile.

\section{Introducción}

La desmembración de la Monarquía hispánica iniciada en la década de 1810 tuvo consecuencias trascendentales en la política latinoamericana del siglo XIX. Entre los problemas más importantes originados tras la Independencia de España se encontraban la formación y consolidación de un Estado autónomo, el afianzamiento del republicanismo como sistema de gobierno y, finalmente, la construcción de la nación como nueva categoría identitaria. El propósito de este estudio es analizar esta última temática para el caso chileno, estudiando el "estado de la cuestión" relativo a la nación, al nacionalismo y la identidad nacional durante aquella centuria, examinando los debates centrales, los aportes más destacados, las limitaciones teóricas y las posibilidades metodológicas que ofrece la historiografía para abordar estos problemas. El balance es pertinente, puesto que la creciente producción de las ciencias sociales en general, y de la historiografía en particular, hacen necesaria una evaluación crítica de las tendencias, enfoques, problemas y metodologías en diálogo en la escena intelectual chilena. Es la invitación de las páginas que siguen.

Una de las primeras constataciones que debemos realizar sobre el problema que nos convoca es la poca atención que los teóricos más destacados en el estudio del nacionalismo (Anthony D. Smith, Ernst Gellner, Elie Kedourie, John Breuilly, Liah Greenfeld, John Hutchinson, Walker Connor y, en menor medida Benedict Anderson) han prestado a América Latina, que ha quedado relegada por lo común a una "nota al pie” (Miller 2006: 203), al ser considerado un caso "anómalo" dentro de los esquemas mas bien eurocéntricos de la cuestión nacional (Hobsbawm 2010: 311). No obstante, esta marginación de América Latina el debate teórico no significa, en modo alguno, que la historiografía americanista haya quedado al margen del estudio del nacionalismo. De hecho, al menos desde mediados de la década de 1990 hay apuestas historiográficas que han invitado a "americanizar" los debates sobre el nacionalismo, según la expresión de Pamplona y Doyle (2008). Este proceso ha intentado consolidarse institucionalmente, cuando a inicios del 2000 se fundó ARENA (Association for Research of Ethnicity \& Nationalism in the Americas), patrocinado por The Richard Walker Institute, University of South Carolina, que ha promovido congresos y publicaciones desde esta perspectiva.

A pesar de este promisorio panorama general, la preocupación por el caso chileno ha ocupado un lugar relativamente marginal dentro del interés de los estudiosos americanistas. Una explicación para esta situación, desde un plano hipotético, se debe a un fenómeno interesante por su dimensión paradójica: al parecer el nacionalismo chileno en el siglo XIX, y a 
diferencia de lo acontecido en otras partes del continente, tuvo relativamente más “éxito”, perspectiva por cierto acentuada en la autopercepción chilena de ser la “excepción” política en América Latina (San Francisco 2009; Jocelyn-Holt 2005), en el sentido de que con una población mayoritariamente mestiza, con un marcado centralismo político, con una relativa estabilidad institucional y con triunfos en guerras internacionales, el proceso de consolidación nacional fue más rápido -pero no por eso sin sobresaltos- que en el resto del continente. Por eso, tal vez, el menor interés de la historiografía latinoamericanista por Chile y centrarse en cambio en otras regiones donde aquel proceso hubiese tenido aparentemente más resistencias (como México, Perú y Brasil). Precisamente ese “éxito” encubre mucho de un proceso complejo y tensionado de construcción nacional, ignorado hasta unos cuantos años.

Sin embargo, este desinterés parece haber cambiado en los últimos veinte años, donde el estudio del nacionalismo en particular y la identidad nacional en general ha cobrado nuevo auge en las ciencias sociales. Las publicaciones relativas a problemas vinculados directamente con el nacionalismo han tenido, como observa un autor, un crecimiento verdaderamente exponencial en esta última década (Özkirimli 2010). Esta renovación se debe a varios fenómenos contextuales globales importantes: la desintegración del bloque soviético y la explosión del problema de las nacionalidades en los Balcanes evidentemente pusieron al nacionalismo en el centro de la reflexión de las ciencias sociales; la recepción en América Latina de los principales trabajos teóricos referentes al nacionalismo -desde 1990 en adelante-, y la superación en la historiografía chilena de una concepción del nacionalismo vinculada a la ideología partidista y autoritaria (hegemónica, sin duda, en el período del Régimen Militar, como quedó planteada ya en 1974 con la Declaración de Principios de la Junta de Gobierno) posibilitada por el regreso a la democracia, permitieron el retorno del nacionalismo como problema histórico digno de ser investigado por la historiografía.

El renovado interés de la historiografía para estudiar el problema de la nación en el siglo XIX dice relación no solo con estos cambios a nivel general, sino también por el giro interpretativo que se dio hacia 1990 para estudiar el período de la Independencia. Gracias a los trabajos de la nueva historia política, se ha permitido superar lo que François-Xavier Guerra ha denominado el esquema "nacionalitario" (1999: 45), que suponía teleológicamente que 1810 era la consecuencia política necesaria de nacionalidades preexistentes, en un esquema de interpretación tradicional del siglo XX (véase por ejemplo Vial Correa 1966). Así, la nación en tanto identificación colectiva- era un fenómeno medianamente resuelto en el siglo XIX, de ahí la ausencia de interés por investigar este problema que no era entendido como tal. Las nuevas investigaciones han cambiado radicalmente este eje interpretativo, proponiendo que antes que una dificultad resuelta por la Independencia, la nación es una de los grandes y complejos problemas por definir, construir, imaginar y socializar en el siglo XIX. 
En síntesis, este replanteamiento desde diversos ángulos del nacionalismo permitió que la historiografía tomase distancia crítica frente al objeto de estudio -la nación- pensándola no ya desde una lógica esencialista, sino como una compleja construcción política, social, cultural, simbólica, discursiva y aún estética, en constante transformación y replanteamiento a través del tiempo. En este sentido, la historiografía ha comenzado a pensar la nación fuera de las lógicas y las categorías del nacionalismo, lo que le ha permitido afinar los análisis y aumentar el espectro de problemas a estudiar. Esto último se debe, además, a una evidente ampliación en la comprensión del nacionalismo como problema histórico de múltiples manifestaciones en la vida social. Como ha señalado Anthony D. Smith (2004: 20), existen al menos cinco caracterizaciones del nacionalismo que al momento se utilizan, con menor y mayor frecuencia, en el campo de las ciencias sociales, lo que evidencia su pluralidad intrínseca del problema a analizar: como un proceso de formación de las naciones; un sentimiento o consciencia de pertenencia a la nación; un lenguaje y un simbolismo de la nación; un movimiento político-social en nombre de la nación; y, finalmente, como una ideología acerca de la nación.

En lo que sigue, consideraremos cómo la historiografía ha analizado estas dimensiones del nacionalismo para el caso chileno de la centuria decimonónica, poniendo atención a diferentes nudos problemáticos donde se han producido los debates más interesantes y los aportes más destacados en lo referente a la construcción de la nación y la identidad nacional, tales como: el rol que ha jugado el Estado en este proceso, la relación entre la identidad nacional y los sectores populares, y, finalmente, el papel de la cultura en la construcción de los imaginarios nacionales.

\section{El Estado y la nación: debates y limitaciones}

En un importante libro publicado a inicios de la década de 1980, el historiador Mario Góngora señalaba escuetamente que el Estado había forjado la nacionalidad chilena (2003: 71-73). Aún cuando Góngora no se explayó mayormente sobre su aserto, éste ha tenido, sorprendentemente, una notable influencia en la historiografía chilena al presentar un punto de partida canónico para el análisis de la nación, subsumiendo este último dentro de las lógicas de la institucionalidad estatal. De hecho, la influencia del ensayo de Góngora ha sido tal que incluso autores que ponen en tela de juicio el real peso del Estado chileno decimonónico, como Alfredo JocelynHolt, han suscrito sin obstáculo a su tesis del Estado como matriz de la nación (1998: 42-43).

Sin desconocer la importancia del Estado en este proceso, particularmente en el siglo XIX donde construir la nación fue uno de sus objetivos más patentes, la postura de Góngora peca de cierta perspectiva metafísica en el planteamiento del Estado (haciéndose eco de Edmund Burke, afirmaría que el Estado “debe ser considerado con reverencia; porque no es una sociedad sobre cosas al servicio de la gran existencia animal, de naturaleza 
transitoria y perecedera. Es una sociedad sobre toda ciencia; una sociedad sobre todo arte; una sociedad sobre toda virtud y perfección”), obviando todo lo de tensionado, complejo y precario tuvo su consolidación institucional y económica en el siglo XIX. Por lo demás, y esta me parece que es una crítica más importante en lo que a la construcción de la identidad nacional se refiere, hay un claro sesgo de unidireccionalidad en la tesis de Góngora sobre este fenómeno, presuponiendo que el Estado creó sin negociación social y simbólica la nacionalidad, asumiendo a priori que la sociedad recibió pasiva y acríticamente de los discursos nacionalizadores de la dirigencia estatal. Como veremos más adelante, ambos planteamientos que se desprenden de la tesis de Góngora han sufrido un interesante cuestionamiento y complejización desde la investigación monográfica.

Sin embargo, Góngora acierta en señalar algunos problemas en que se puede evidenciar la creación estatal de la nación, como en la educación, las fiestas nacionales y los símbolos (2003: 72). Es precisamente en estas tres dimensiones en que se han producido en las últimas décadas aportes relevantes para la comprensión de construcción de la identidad nacional chilena. Comencemos por la educación, que ha sido objeto de una particular atención de la historiografía, tanto para el siglo XIX como para inicios del XX. Inicialmente, como señala Sol Serrano, la educación fue pensada explícitamente desde la intelectualidad y la dirigencia estatal como un modo de socializar tanto los principios republicanos como "forjar una nación con una identidad común a todos los habitantes de un territorio, es decir, forjar una ideología nacional como fuente de legitimación política” (1994: 64). Este proceso, como bien ha notado recientemente Ricardo Iglesias, sufrió una aceleración en la segunda mitad del siglo XIX, lo que fue, hasta cierto punto, el correlato del afianzamiento del poder estatal sobre el territorio nacional. Si en 1852 la matrícula de alumnos de educación básica era de 23.504, hacia 1900 la cantidad ascendía a 163.792 alumnos, evidenciando el papel protagónico de la educación como plataforma de diseminación masiva de la identidad nacional (2009: 67-69). Lo cierto es que, como han apuntado estudios recientes, la educación también fue una herramienta crucial para culturizar en clave nacional a poblaciones pluriétnicas que no estaban en los límites originales del Estado chileno decimonónico, pero que tras su expansión hacia el norte tras la Guerra del Pacífico y hacia el sur con la anexión militar de la Araucanía quedaron insertas en el radio de acción estatal. En este sentido, la chilenización por las aulas fue un fenómeno clave tanto en lo que dice relación con la población aymara y peruana en el norte del país (González Miranda 2002), como con la etnia mapuche al sur del Bío-Bío (Donoso 2008). Lo interesante es que la expansión territorial chilena hacia el norte como al sur coincidió, desde la década de 1880, con un período de reformas en la educación, destinadas a fomentar la educación cívica y la difusión de valores patrióticos, proceso que se extendió y consolidó en las primeras décadas del siglo XX, como lo ha evidenciado la historiografía reciente (Rojas Flores 2004).

Otra dimensión en que el Estado tuvo un papel relevante en la construcción de la identidad nacional tiene que ver con los aspectos simbólicos 
y rituales. La importancia de los símbolos para la construcción nacional radica en que, como ha notado Karen Cerullo, estos son tótems modernos, signos que encarnan la identidad que buscan representar, al tiempo que distinguen y reafirman la identidad de la comunidad representada frente a los “otros". Himnos, banderas y escudos cumplen esta función en el mundo de las naciones modernas (1993: 244). Un reciente trabajo desde esta línea por Trinidad Zaldívar y Macarena Sánchez (2009) analiza la construcción de símbolos de identidad en la República temprana, tales como banderas y escudos, aunque también adentrándose en el espacio de las fiestas cívicas. Estas últimas, como bien ha examinado Paulina Peralta (2007) en su estudio sobre las primeras conmemoraciones nacionales (12 de febrero, 5 de abril y 18 de septiembre) fueron una de las instancias claves de socialización tanto del republicanismo como de la identidad nacional. Por cierto, estas instancias festivas no fueron las únicas, pues tras la Guerra contra la Confederación la conmemoración de la batalla de Yungay fue importante para socializar en la población ideas-fuerza en torno a la identidad nacional, como la de Chile como un país guerrero (Cid 2008).

Por cierto, la guerra en tanto instancia límite catalizadora de nacionalismo ha recibido también la atención de la historiografía reciente. Analizando los conflictos bélicos desde una perspectiva sociocultural Gabriel Cid (2011) y Carmen Mc Evoy (2006a; 2000; 2011) han enfatizado el estrecho vínculo generado en el siglo XIX entre las guerras y la construcción de la nación chilena. Esto no solo por elementos tales como el reclutamiento masivo y la centralización del poder que toda guerra provoca, sino también desde la perspectiva de la historia cultural y de los imaginarios. Así, se ha prestado especial atención no solo a los discursos patrióticos forjados a partir de estas instancias para legitimar la postura del Estado-nacional en los conflictos, sino también la materialización del imaginario bélico en el espacio de la sociedad civil, por medio de prácticas rituales y simbólicas.

El valor de las instancias rituales y los símbolos radica en que pueden cooptar nacionalmente a la población ajena a la cultura alfabetizada, como lo fue en su mayoría la población decimonónica. Este dificultad en el proceso de construcción nacional que se intentó superar por la vía de la educación, aunque no fue un óbice para la socialización de la identidad por otros canales. En este sentido, el trabajo pionero de Rafael Pedemonte (2008) sobre los himnos patrióticos y la identidad nacional resulta clave, pues analiza precisamente un importante medio de difusión de la nacionalidad en una población mayoritariamente oral. Lo interesante de los trabajos de Peralta y Pedemonte es que ambos explicitan el protagónico papel de los sectores populares en decodificación y resignificación de los símbolos oficiales, complejizando la tesis de una creación unidireccional y sin tensiones de la identidad desde la estructura estatal, al proponer como parte central de las investigaciones en torno al nacionalismo la relevancia que juega el problema de la recepción de los discursos.

En este sentido, y como bien ha destacado Fernando Purcell (2006), es necesario prestar atención tanto a los mecanismos de socialización de la 
identidad nacional como su grado de efectividad e impacto en la población, dado que no hay una correspondencia necesaria ni instantánea entre la intencionalidad estatal y la eficacia social de sus propuestas. Debido a esto, es preciso examinar tanto la tensión entre prácticas y actores, como entre propósitos oficiales y agentes mediadores en el proceso de difusión de la identidad nacional. Así, Andrés Estefane (2004) ha evidenciado, desde una perspectiva bastante novedosa, la resistencia de la población a los censos (pensados como un medio de medir, racionalizar y homogeneizar una población heterogénea), así como la necesidad del Estado de contar con las redes de poder local para llevar a cabo sus propósitos. De ahí que consideremos que uno de los conceptos que puede contribuir a avanzar hacia nuevas perspectivas de estudio es el de negociación cultural, ya que no solo rompe la undireccionalidad de los análisis basados solo en la institucionalidad estatal, sino porque también permite incorporar e integrar en las explicaciones a nuevos actores, cuyas voces y prácticas han sido marginadas constantemente de los análisis de la historiografía tradicional.

\section{La nación y el mundo popular}

Como hemos analizado, la historiografía reciente ha prestado cada vez más atención a la recepción de los discursos nacionalizadores, especialmente en el mundo popular. Si, como nota Eric Hobsbawm las naciones se construyen dualmente, con un mayor énfasis “desde arriba” -en referencia al aparato estatal-, estas no pueden entenderse cabalmente si no se analizan también "desde abajo", es decir, cómo las personas comunes asimilan o no los discursos nacionalistas (2004: 18-19). Desde esta perspectiva, en el último tiempo se puede evidenciar un importante cuestionamiento historiográfico a la tesis de la creación unidireccional de la nación por parte del Estado. Así, por ejemplo, en un libro indispensable, Julio Pinto y Verónica Valdivia (2009) han analizado este problema en el período fundacional de la nación chilena (1810-1840), examinando los intentos de movilización nacional de los sectores populares por parte de los sectores dirigentes y la indiferencia y resistencia de los primeros frente a estos intentos. Tal cooptación nacional -en una dimensión simbólica y no ciudadana- sólo se verificaría, de acuerdo a estos autores, tras la Guerra contra la Confederación.

Para un período posterior, en el último tercio del siglo XIX, la historia social también ha abordado de manera muy valiosa la perspectiva de la nación vista “desde abajo”. En un estudio pionero Julio Pinto analizó los discursos populares en el mundo pampino durante la Guerra del Pacífico, donde se manifestó una suerte de "patriotismo popular" de amplia difusión entre sus pares sociales debido a la movilidad espacial de los peones chilenos, "patriotismo" que, a medida que avanzó el siglo, se fue tensionando entre las identidades de clase y nacional (Pinto 1997; Pinto, Valdivia y Artaza 2003). Lo interesante del trabajo de Pinto, y esto es importante destacarlo como una de las reflexiones importantes para una estructuración del análisis de la nación "desde abajo", es que salva la antinomia de la "clase" 
como opuesta a la "patria”, tan propia de la historiografía marxista. También lo es la antinomia opuesta, de marcado acento nacionalista, para lo cual la identidad nacional está por sobre cualquier otra forma de identificación. A estas alturas del debate en las ciencias sociales, es claro que la identidad nacional no es exclusiva ni excluyente de otros tipos de identificaciones, por más que el nacionalismo quiera hacer de ésta la identidad hegemónica: la identidad nacional es solamente una de muchas identidades -como las de clase, religiosa, regional, género, generacional, entre muchas otras- que coexisten en cada persona y grupo (Smith 1993: 3-8).

A pesar de lo dificultoso que resulta documentar la visión de la nación desde el mundo popular, particularmente en el siglo XIX, trabajos recientes como los de Maximiliano Salinas (2004) y Pamela Tala (2001) sobre los escritores populares Juan Rafael Allende y Rosa Araneda, respectivamente, permiten entender ciertas lógicas de articulación de los significados de la identidad nacional en clave popular, muchas veces discordantes de los discursos oficialistas sobre lo nacional, y a veces en abierta crítica contra estos. En una línea algo distinta, aunque también interesada en rescatar la mirada desde la nación “desde abajo”, particularmente en una perspectiva de historia política, James A. Wood (2009) ha analizado las articulaciones del nacionalismo popular de la guardia nacional en la primera mitad del siglo XIX, y su relaciones con la opinión pública de la época, en especial en la década de 1840 con la labor del editor popular Santiago Ramos. En síntesis, aunque es sumamente dificultoso acceder a la visión del mundo popular, particularmente en lo que respecta a la identidad nacional, estos trabajos evidencian lo valioso que resulta y la complejidad que adquiere este problema al analizar la perspectiva de la nación “desde abajo”, lo que, cuantitativa y cualitativamente, sigue siendo la gran deuda pendiente de la historiografía sobre la construcción de nación en el siglo XIX chileno.

Otra forma de estudiar este problema, y que ha producido estimulantes resultados, es analizarlo no tanto desde soportes documentales escritos -que sin duda no dejan de ser un filtro mediatizado para acercarse al “bajo pueblo"-, sino enfatizar la aproximación desde sus prácticas culturales. En este punto, debemos detenernos en una problemática afín que ha ocupado a la historia social en torno a la relación existente entre el mundo popular y la identidad nacional. Si autores como Góngora otorgan al Estado un papel gravitante en la conformación de la nacionalidad, historiadores como Gabriel Salazar invierten esta postura desde la historia social. Para Salazar, y articulando su reflexión dentro del concepto de "cultura popular”, señala que en Chile la cultura popular es autónoma y opuesta a la cultura de la elite. Para Salazar (1991), entonces, la identidad nacional chilena se encuentra solamente en la cultura popular, la única con capacidad creadora. La postura de Salazar, que tiende a esencializar el concepto de pueblo, peca precisamente por su falta de complejización en el análisis de un proceso que la historiografía reciente afortunadamente ha matizado, aunque persiste la tendencia a atribuirle a la cultura popular un esencialismo nacional en contra de las fracasadas propuestas de la elite (Donoso 2009). 
En efecto, no es posible entender en toda su complejidad la "cultura popular" -cuyas cualidades supuestamente distintivas tienen generalmente un sesgo arbitrario por parte del historiador y también de los folkloristas- como un campo autónomo o excluyente de los discursos escritos y elitistas. Sería útil avanzar hacia una comprensión de la "cultura popular" dentro de un campo en permanente negociación, transmisión, resemantización y diferentes formas de apropiación, sin obviar las disputas de poder y la desigualdad de condiciones en que esta se manifiesta (Chartier 1994).

Un aspecto que requiere sin duda mayor reflexión tiene que ver con precisamente pasar desde los sectores populares como meros receptores de los discursos nacionalistas desde las elites político-intelectuales (un proceso que a menudo se describe como pasivo), a una visión desde el mundo popular más bien creativo, inserto como un actor relevante en la circulación de estos códigos nacionalistas. En este sentido, la historiografía debería complejizar su aproximación a los sectores populares, prestando atención al momento en que de receptores pasan a ser demandantes que condicionan e interpelan activamente la producción de discursos plausibles sobre lo nacional, hasta pasar a ser productores y difusores del discurso nacionalista, como aconteció en la segunda mitad del siglo gracias, por ejemplo, a la lira popular. Pero también, debería ser consciente de lo plural que son los sectores populares, tanto por su locación espacial-laboral (rural/ urbano) sino también por sus mismas jerarquías y distinciones internas.

Desde esta perspectiva dialogal, por ejemplo, Gabriel Cid (2009) ha analizado la invención del roto - pensando en el siglo XX como la quintaesencia de la chilenidad- en el siglo XIX, prestando atención a su complejo proceso de reposicionamiento en la opinión pública chilena, donde tanto la elite como los portavoces del mundo popular ayudaron a su cristalización como ícono nacional. En un registro analítico similar, Sebastián Rico (2009) estudia el tensionado proceso de construcción de las chinganas en el siglo XIX, que va desde la exclusión hasta su incorporación en la cultura chilena, muy similar a lo acontecido con la música popular campesina como la zamacueca (Torres 2008).

\section{La nación y sus “otros"}

El mundo popular chileno fue considerado durante buena parte del siglo XIX por la elite como un "otro" al que había que civilizar y moralizar. Esta última situación nos habla también a nivel general sobre cómo se construyen las identidades, sociales o nacionales: la apelación a un "otro" siempre se constituye en un factor decisivo desde donde articular la propia visión, sus supuestas particularidades y diferencias. La identidad se construye, así, en una dialéctica de espejos.

Uno de estos espejos desde los cuales se articuló la identidad nacional en el siglo XIX fue el caso de los mapuche; aunque es importante señalar que este fue un proceso que se vivió de forma transversal en América 
Latina, donde las elites dirigentes echaron mano simbólicamente a sus indígenas para articular la historia mítica de las nacientes naciones (Earle 2008). Para el caso chileno, durante la primera mitad de la centuria, y particularmente en las luchas independentistas los "araucanos" fueron construidos como referentes míticos de identidad, pues permitían otorgarle mayor densidad histórica a la lucha entre patriotas y realistas, como bien notó hace años Simon Collier (1977: 199-204), y cuyas huellas han seguido con lucidez para un período posterior Holdenis Casanova (2000) y Viviana Gallardo, aunque desde una perspectiva más ensayística (2001). Sin duda, el trabajo de Jorge Pinto Rodríguez (2003) emerge como uno de los aportes más destacados y completos a este respecto, ya que detalla en su complejidad el proceso de inclusión inicial de los mapuche en la idea de nación, hasta pasar a la exclusión (simbólica, política y económica) en la segunda mitad del siglo XIX, y las consecuencias de este proceso en el siglo XX.

El caso de los mapuche ejemplifica bien, además, el valor que el concepto de "etnicidad" en la construcciones de las identidad nacionales. En efecto, en la convivencia social de grupos pluriétnicos la catálisis del nacionalismo puede aumentar de forma importante, sobre todo, porque resulta aparentemente más fácil acentuar las diferencias -reales o imaginarias- entre los grupos étnicos. Esto fue particularmente cierto para el caso chileno de la segunda mitad del siglo XIX y comienzos del siglo XX, cuando el encuentro con grupos étnicos diversos contribuyó a delimitar los contornos de lo que se estimó era la nación y cuáles eran sus “otros”. Para comprender estas dinámicas, el trabajo de Jean-Pierre Blancpain (2005) sobre la relación entre la inmigración y el nacionalismo resulta clave, fenómeno que ya había observado el estudio pionero de Carl Solberg (1970), en uno de los primeros trabajos historiográficos, además, sobre el nacionalismo en Chile, aunque también incorpora comparativamente a Argentina, en el período que va de 1890 a 1914.

La compleja e íntima relación entre nacionalismo y etnicidad también queda reflejada en el reciente trabajo de William Skuban (2007) sobre las tensiones nacionalistas en la frontera norte de Chile, donde la incorporación de territorios peruanos y bolivianos a la soberanía estatal chilena en la década de 1880 significó una serie de conflictos raciales producto del proceso de nacionalización, que desembocó, a inicios del siglo XX en movimientos nacionalistas y xenofóbicos violentistas como las Ligas Patrióticas, que ha estudiado muy bien Sergio González Miranda (2004).

\section{La dimensión "cultural-simbólica” de la nación}

El proceso de formación nacional, como ha quedado claro, no está relegado solamente al aspecto político-institucional. El asunto es más complejo, pues en esta construcción estos aspectos se entremezclan con otros factores. En este sentido es útil la distinción de Bárbara Silva, según la cual en los procesos de construcción nacional se concatenan una dimensión "político-discursiva” y una “cultural-simbólica” (2006: 21). Esta última dimen- 
sión pone su acento en los símbolos, ritos, mitos y estereotipos de identificación común. Aquí se han generado una serie de aportes originales relativos al estudio de la identidad nacional en el siglo XIX.

Carlos Sanhueza (2006), por ejemplo ha argumentado lúcidamente sobre la necesidad de enfocar el análisis de la identidad nacional desde la perspectiva de la cultura. En efecto, si se analizan las representaciones discursivas de la identidad que realizaron los viajeros chilenos en Europa en general, y desde Alemania en particular, se puede apreciar cómo esas representaciones de lo nacional, emanadas desde la experiencia de la distancia, se articularon no desde la política, sino que fundamentalmente desde la cultura.

La literatura como soporte discursivo de lo nacional fue una de las temáticas fundamentales de la Generación de 1842, aquella generación de intelectuales claves en la búsqueda de la construcción de una identidad nacional renovada, con fuerte contenido proyectual, republicano y liberal (Stuven 1987). En este punto es necesario destacar dos aspectos relacionados con esta generación de intelectuales. En primer término, las polémicas del lenguaje y su vinculación con la nación (Narvaja de Arnoux 2008) y la búsqueda de referentes literarios capaces de plasmar las particularidades nacionales. Así, a mediados del siglo XIX, como ha estudiado Bernardo Subercaseaux (1981), pueden constatar debates e intentos por plasmar literariamente tanto tópicos chilenos como un estilo propiamente nacional. La obra de Alberto Blest Gana Martín Rivas fue una expresión clara de esta búsqueda, constituyéndose para la literatura chilena en lo que Doris Sommer denomina "ficciones fundacionales" (2004: 263-280).

Otra de las temáticas surgidas con la Generación de 1842 y que tuvo desarrollos a lo largo del siglo fue el de la función social de la historia: esta disciplina fue puesta en el centro del debate como uno de los medios conformantes de la identidad nacional. El trabajo pionero de Allen Woll (1982) ha analizado detalladamente el papel de la historia en el siglo XIX chileno, examinando cómo ésta fue utilizada como herramienta para las contiendas políticas, para dirimir los conflictos diplomáticos del Estado y, especialmente, como una forma de socializar la identidad nacional en las aulas chilenas. En cuanto a obras historiográficas específicas y su impacto en la construcción nacional, Rafael Sagredo (2007) ha examinado la elaboración de la primera historia oficial de Chile, la Historia física y política de Chile, elaborada por el científico francés Claudio Gay por encargo expreso del gobierno; mientras que la historiadora norteamericana Gertrude Yaeger (1981) ha estudiado la monumental Historia General de Chile de Diego Barros Arana a la luz de su contexto de producción y su filiación con la identidad nacional.

Los productores de sentido nacional del pasado asumieron un rol fundamental en el siglo XIX, con una marcada autopercepción de su rol social, visualizado como un verdadero "sacerdocio cívico". Benjamín Vicuña Mackenna, por ejemplo, con su serie de biografías ayudó a configurar 
un panteón heroico nacional y forjar así la imagen de los “padres de la patria” (Vicuña 2009). Lo cierto es que la historiografía se ha desligado aunque muy recientemente- de la imagen canónica de los "padres de la patria”, mirando con mayor criticismo este fenómeno, enfocándose más bien en los complejos procesos políticos, sociales y culturales que subyacen a estas construcciones heroicas. Mucho de esta renovación se la debemos al lúcido trabajo fundacional de William F. Sater, quien a inicios de la década de 1970 publicó su innovadora y polémica obra relativa al proceso de heroificación colectiva de Arturo Prat, analizando las oscilaciones en su culto tras su muerte (1973).

El problema de la construcción de un panteón nacional ha sido retomado actualmente a través de otros análisis. Así, Carmen Mc Evoy ha estudiado la construcción de Bernardo O’Higgins como el "Padre de la Patria” en el imaginario nacional a través del estudio de su funeral de Estado (2006b); mismo escenario desde el cual Ana María Stuven (2006) ha analizado las tensiones para la inclusión/exclusión del panteón republicano de figuras ajenas a la hegemónica tradición católica, como José Miguel Infante, en contraposición a la imagen de Andrés Bello; a la vez que Alejandro San Francisco (2006) se ha detenido en los funerales de José Manuel Balmaceda como un momento clave en el proceso de reconciliación política tras los estragos de la Guerra Civil de 1891. La imagen del presidente Balmaceda ha sido examinada también por Rodrigo Mayorga (2008), quien ha analizado el proceso de reposicionamiento de su figura tras 1891, desde su descalificación hasta su heroificación, enfocándose la apropiación de su imagen en pos de legitimidad política de los partidos afines a su ideario.

Dentro de la dimensión “cultural-simbólica” de la nación resulta importante destacar novedosas líneas de análisis. Una de ellas es el estudio del territorio y su impacto en la formación del imaginario nacional, donde la serie de trabajos de Rafael Sagredo (1998; 2005; 2008; 2009) son una referencia obligada Lo cierto es que la representación científica y cartográfica del territorio fue de la mano con la necesidad de reelaborar estéticamente el espacio geográfico hasta transformarlo en paisaje. A pesar de que Isabel Cruz (2000) se ha aproximado a su estudio, esta es una temática sobre la cual queda mucho por trabajar, en especial si se considera la importancia que tuvo en la conformación identitaria la noción de Chile como “copia feliz del Edén” (Cid y Vergara, 2011). Esta idealización de las bondades de la naturaleza nacional pronto se vinculó con la necesidad del Estado de resguardar aquellas singularidades botánicas, minerales y zoológicas, para lo cual la instauración de un Museo fue clave para lograr esta representación de lo chileno (Schell 2001). La ciencia, como se ve, tuvo un papel protagónico en la conformación de representaciones sobre lo nacional, como lo han evidenciado trabajos recientes como el de Zenobio Saldivia (2009) y Stefanie Gänger (2009), quien en una línea de análisis bastante original estudia las filiaciones entre la arqueología y el nacionalismo a fines del siglo XIX en la frontera chileno-peruana. 
Lo chileno, no obstante, también debía ser representado en el arte, lo cual siempre supuso interpretaciones polémicas sobre qué era lo que constituía lo característicamente identitario y como debería ser su puesta en escena (De la Maza 2010). Las creaciones artísticas en el siglo XIX eran pensadas como una forma de exhibir el prestigio del Estado y visualizar lo que se creía eran las diversas aristas de lo nacional, para la cual las exhibiciones y la puesta en marcha de un Museo de Bellas Artes fueron claves en este proceso (Schell 2009; Hernández 2006). Teniendo en mente este marco referencial, recientemente se ha comenzado a dar un bienvenido giro desde la historia del arte a la historia de las representaciones de la identidad desde el arte. Jacinta Vergara (2009), por ejemplo, ha analizado con agudeza la representación de lo chileno en la pintura de artistas como el bávaro Johann Moritz Rugendas. En una línea similar, aunque en un período más amplio -pues incluye las obras de Gil de Castro y Rugendas- lo ha hecho Claudia Borri (2008); mientras que recientemente Gabriel Cid ha estudiado las relaciones entre pintura de historia y la construcción de los imaginarios nacionales, a propósito del caso de la Guerra del Pacífico (2011). El arte, sin embargo, no solamente estuvo relegado al espacio de los museos, sino que se apropió de los espacios públicos en una nacionalización de las ciudades tanto por medio de la toponimia urbana como a través de la construcción de monumentos vinculados a la conformación de una memoria histórica nacional (Voionmaa 2004; Salgado 2010).

\section{Conclusiones}

Como hemos analizado sintéticamente en estas páginas, la historiografía chilena referente al nacionalismo y la identidad nacional ha sido particularmente ingente en los últimos veinte años, concordante con el creciente interés que a nivel global las ciencias sociales han otorgado al nacionalismo. Considero que tal explosión se ha debido a una mayor complejización y criticismo en el enfoque de la nación, sumado a una desvinculación del nacionalismo entendido como mera ideología partidista y pro-fascista, aunque claramente esto no significa que en la actualidad grupos extremistas vinculados en general al movimiento neonazi sigan comprendiendo el concepto bajo esas lógicas (Caro 2007)

Sin embargo, las nuevas perspectivas historiográficas sobre el nacionalismo reflejan no solo la importancia de este problema en el siglo XIX, sino además su inherente diversidad histórica dadas sus múltiples plasmaciones en la vida social, política y cultural, que hicieron de la nación un concepto esencialmente polisémico y lo tornaron en un campo de significados en disputa en aquella centuria . Como ha analizado recientemente Ana María Stuven (2009), la nación se fue complejizando a medida que avanzaba el siglo XIX, pasando desde dimensiones institucionales, culturales hasta desembocar, a fines del siglo, en el problema de la efectiva inclusión social y política de los sectores más postergados. La nación se hizo más densa históricamente -como mito y problema- y también más amplia al intentar agrupar en su seno a la totalidad de los habitantes de 
Chile. La emergencia de la clase media a inicios del siglo XX tanto como productora y receptora de los discursos nacionalistas no hizo sino complejizar aún más este problema, como ha notado Patrick Barr-Melej (2001).

Esta diversidad, densidad y complejidad de la nación en el siglo XIX no solo permite y requiere aproximaciones desde distintas áreas historiográficas, que van desde la historia política, social, cultural, intelectual, pero en una integración más bien dialogal. Además, y esto también resulta evidente dada la múltiple producción historiográfica analizada en estas páginas, es preciso no solamente afinar los conceptos claves desde los cuales se articulan los trabajos, dotando de mayor elasticidad a construcciones conceptuales que inducen a lecturas unidireccionales y monolíticas -y por lo mismo esquemáticas y poco comprensivas- de los procesos, sino que también hacen necesaria la reflexión y el diálogo multidisciplinar con la sociología, la ciencia política, la filosofía, la literatura y los estudios culturales. Teniendo en mente este escenario, la tarea de la historiografía sobre la construcción nacional es más ardua que nunca. 


\section{Bibliografía}

Barr-Melej, Patrick (2001), Reforming Chile. Cultural politics, nationalism and the rise of the middle class. Chapel Hill: University of North Carolina Press.

Blancpain, Jean-Pierre (2005), Immigration et nationalisme au Chili 18101925: Un pays à l'écoute de l'Europe. Paris: L'Harmattan.

Borri, Claudia (2008), “La formación de la identidad nacional chilena a través de la pintura de José Gil de Castro y de Mauricio Rugendas”, en Inmaculada Rodríguez (ed.), Arte, poder e identidad en Iberoamérica: de los virreinatos a la construcción nacional. Castelló: Universitat Jaume I.

Caro, Isaac (2007), Extremismos de derecha y movimientos neonazis: Berlín, Madrid, Santiago. LOM, Santiago.

Casanova, Holdenis (2000), “Entre la ideología y la realidad: la inclusión de los mapuche en la nación chilena (1810-1830)”, Revista de Historia Indígena, $\mathrm{N}^{\circ} 4$.

Cerullo, Karen (1993), "Symbols and the world system: national anthems and flags”, Sociological Forum, Vol. 8, N².

Chartier, Roger (1994), “ 'Cultura popular': retorno a un concepto historiográfico”, Manuscrits, $\mathrm{N}^{\circ} 12$.

Cid, Gabriel (2008), “Nacionalizando la ‘segunda independencia’ chilena. Fiestas y discursos cívico-religiosos en torno a la Guerra contra la Confederación, 1836-1851”, Bicentenario. Revista de Historia de Chile y América, Vol. 7, $\mathrm{N}^{\circ} 2$.

Ídem (2009), “Un ícono funcional: la invención del roto como símbolo nacional, 1870-1888”, Gabriel Cid y Alejandro San Francisco (eds.), Nación y nacionalismo en Chile. Siglo XIX. Centro de Estudios Bicentenario, Santiago, Vol. 1.

Ídem (2011), La Guerra contra la Confederación. Imaginario nacionalista y memoria colectiva en el siglo XIX chileno. Ediciones Universidad Diego Portales, Santiago.

Ídem (2011), “Arte, guerra e imaginario nacional: la Guerra del Pacífico en la pintura de historia chilena, 1879-1912”, en Carlos Donoso y Gonzalo Serrano (eds.), Chile y la Guerra del Pacífico. Universidad Andrés Bello/ Centro de Estudios Bicentenario, Santiago.

Ídem y Vergara, Jacinta (2011), “Representando la 'copia feliz del Edén'. Rugendas: paisaje e identidad nacional en Chile, siglo XIX”, Revista de Historia Social y de las Mentalidades, Vol. 15, № 2. 
Collier, Simon (1977), Ideas y política de la independencia chilena, 18081833. Andrés Bello, Santiago.

Cruz, Isabel (2000), “¿Arcadia en el confín del mundo? El paisaje romántico en Chile en la pintura de los artistas viajeros (1829-1850)”, en Vida rural en Chile durante el siglo XIX. Academia Chilena de la Historia, Santiago.

De la Maza, Josefina (2010), "Por un arte nacional. Pintura y esfera pública en el siglo XIX chileno”, en Rafael Sagredo (ed.), Ciencia-Mundo. Orden republicano, arte y nación en América. Centro de Investigaciones Diego Barros Arana/ Editorial Universitaria, Santiago.

Donoso, Andrés (2008), Educación y nación al sur de la frontera. Organizaciones mapuche en el umbral de nuestra contemporaneidad, 1880-1930. Pehuén, Santiago.

Donoso, Karen (2009), “Fue famosa la chingana...” Diversión popular y cultura nacional en Santiago de Chile, 1820-1840”, Revista de Historia Social y de las Mentalidades, Vol. XIII, $\mathrm{N}^{\circ} 1$.

Earle, Rebecca (2008), The return of the native. Indians and myth-making in Spanish America, 1810-1930. Duke University Press, Durham.

Estefane, Andrés (2004), “ ‘Un alto en el camino para saber cuántos somos'... Los censos de población y la construcción de lealtades nacionales. Chile, siglo XIX”, Historia, Vol. 37, N 1.

Gallardo, Viviana (2001), “Héroes indómitos, bárbaros y ciudadanos chilenos: el discurso sobre el indio en la construcción de la identidad nacional”, Revista de Historia Indígena, № 5.

Gänger, Stefanie (2009), “Conquering the past: post-war archaeology and nationalism in the borderlands of Chile and Peru, c.1880-1920", Comparative Studies in Society and History, Vol. 51, N 4.

Góngora, Mario (2003 [1 $1^{\mathrm{a}}$ ed. 1982]), Ensayo histórico sobre la noción de Estado en Chile en los siglos XIX y XX. Editorial Universitaria, Santiago.

González Miranda, Sergio (2002), Chilenizando a Tunupa. La escuela pública en el Tarapacá andino, 1880-1990. DIBAM, Santiago.

Ídem (2004), El dios cautivo. Las Ligas Patrióticas en la chilenización compulsiva de Tarapacá (1910-1922). LOM, Santiago.

Guerra, François-Xavier (1999), "De lo uno a lo múltiple: dimensiones y lógicas de la Independencia”, en Anthony McFarlane y Eduardo Posada Carbó (eds.), Independence and revolution in Spanish America: perspectives and problems. ILAS, Londres. 
Hernández, Carmen (2006), “Chile a fines del siglo XIX: Exposiciones, museos y la construcción del arte nacional”, en Jens Andermann y Beatriz González-Stephan (ed.), Galerías del progreso: Museos, exposiciones y cultura visual en América Latina. Beatriz Viterbo Editora, Rosario.

Hobsbawm, Eric (2004), Naciones y nacionalismo desde 1780. Crítica, Barcelona.

Ídem (2010), “Nacionalismo y nacionalidad en América Latina”, en Pablo Sandoval (comp.), Repensando la subalternidad. Miradas críticas desde/ sobre América Latina, Instituto de Estudios Peruanos, Lima.

Iglesias, Ricardo (2009), “El papel de la educación en la construcción del Estado nacional chileno en el siglo XIX”, en Gabriel Cid y Alejandro San Francisco (eds.), Nación y nacionalismo en Chile. Siglo XIX. Centro de Estudios Bicentenario, Santiago, Vol. 2.

Jocelyn-Holt, Alfredo (2005), “¿Un proyecto nacional exitoso? La supuesta excepcionalidad chilena”, en Francisco Colom González (ed.), Relatos de Nación. La construcción de las identidades nacionales en el mundo hispánico. Iberoamericana/Vervuert, Madrid.

Ídem (1998), El peso de la noche. Nuestra frágil fortaleza histórica. Planeta, Santiago.

Mayorga, Rodrigo (2008), “Un héroe para el partido, un héroe para la nación. Balmaceda y su imagen ante la historia. Los años formativos (18911897)", en Rodrigo Mayorga (ed.), Lejos del ruido de las balas. La guerra civil chilena de 1891. Centro de Estudios Bicentenario, Santiago.

Mc Evoy, Carmen (2000), “ 'Bella Lima ya tiemblas llorosa del triunfante chileno en poder’: una aproximación a los elementos de género en el discurso nacionalista chileno”, en Narda Henríquez (comp.), El hechizo de las imágenes. Estatus social, género y etnicidad en la historia peruana. Pontificia Universidad Católica del Perú, Lima.

Ídem (2006), “ 'De la mano de Dios’. El nacionalismo católico chileno y la Guerra del Pacífico, 1879-1881”, Bicentenario. Revista de Historia de Chile y América, Vol. 5, № 1 .

Ídem (2006), “El regreso del héroe: Bernardo O’Higgins y su contribución en la construcción del imaginario nacional chileno, 1868-1869”, en Carmen Mc Evoy (ed.), Funerales republicanos en América del Sur: tradición, ritual y nación, 1832-1896. Centro de Estudios Bicentenario, Santiago.

Ídem (2010), Armas de persuasión masiva. Retórica y ritual en la Guerra del Pacífico. Centro de Estudios Bicentenario, Santiago. 
Ídem (2011), Guerreros civilizadores. Política, sociedad y cultura en Chile durante la Guerra del Pacífico, Ediciones Universidad Diego Portales, Santiago.

Miller, Nicola (2006), "The historiography of nationalism and national identity in Latin America”, Nations and Nationalism, Vol. 12, № 2.

Narvaja de Arnoux, Elvira (2008), Los discursos sobre la nación y el lenguaje en la formación del Estado (Chile, 1842-1862). Estudio glotopolítico. Santiago Arcos/SEMA, Buenos Aires.

Özkirimli, Omut (2010), Theories of nationalism. A critical introduction. Palgrave Macmillan, New York.

Pamplona, Marco Antonio y Doyle, Don H., org. (2008), Nacionalismo no novo mundo. A formação de estados-nação no seculo XIX. Record, Rio de Janeiro.

Pedemonte, Rafael (2008), Los acordes de la patria. Música y nación en el siglo XIX chileno. Globo Editores, Santiago.

Peralta, Paulina (2007), ¡Chile tiene fiesta! El origen del 18 de septiembre (1810-1837). LOM, Santiago.

Pinto Rodríguez, Jorge (2003), La formación del Estado y la nación, y el pueblo mapuche. De la inclusión a la exclusión. DIBAM, Santiago.

Pinto, Julio y Valdivia, Verónica (2009), ¿Chilenos todos? La construcción social de la nación (1810-1840). LOM, Santiago.

Pinto, Julio (1997), “¿Patria o clase? La Guerra del Pacífico y la reconfiguración de las identidades populares en el Chile contemporáneo”, Contribuciones Científicas y Tecnológicas, Área Ciencias Sociales y $\mathrm{Hu}$ manidades, $\mathrm{N}^{\circ} 116$.

Pinto, Julio; Valdivia, Verónica y Artaza, Pablo (2003), "Patria y clase en los albores de la identidad pampina (1860-1890)”, Historia, № 36.

Purcell, Fernando (2006), "Discursos, práticas e atores na construção do imaginário nacional chileno (1810-1850)”, en Marco A. Pamplona y Maria Elisa Mäder, (eds.), Revoluções de independências e nacionalismos nas Américas. Região do Prata e Chile. Paz e Terra, São Paulo, Vol. 1.

Rico, Sebastián (2009), “De lo popular a lo nacional en el Chile decimonónico: la chingana como un espacio de encuentro y diferenciación en torno a la nación”, en Gabriel Cid y Alejandro San Francisco (eds.), Nación y nacionalismo en Chile. Siglo XIX. Centro de Estudios Bicentenario, Santiago, Vol. 2. 
Rojas Flores, Jorge (2004), Moral y prácticas cívicas en los niños chilenos, 1880-1950. Ariadna, Santiago.

Sagredo, Rafael (2008), "Chile: de fines terrae imperial a 'copia feliz del edén’ autoritario”, en José Carlos Chiaramonte, Carlos Marichal y Aimer Granados (comp.), Crear la nación. Los nombres de los países de América Latina. Sudamericana, Buenos Aires.

Ídem (2005), “Claudio Gay y la representación de Chile”, en Alejandra Araya, Azun Candina y Celia Cussen (eds.), Del nuevo al viejo mundo: mentalidades y representaciones desde América. Facultad de Filosofía y Humanidades Universidad de Chile, Santiago.

Ídem (2007), “De la historia natural a la historia nacional. La historia física y política de Claudio Gay y la nación chilena”, en Claudio Gay, Historia física y política de Chile. Historia. Cámara Chilena de la Construcción/DIBAM/PUC, Santiago, tomo 1.

Ídem (2009), “Geografía y nación. Claudio Gay y la primera representación cartográfica de Chile”, Estudios Geográficos, Vol. LXX, º 266.

Ídem (1998), “La idea geográfica de Chile en el siglo XIX”, Mapocho, N 44.

Salazar, Gabriel (1991), “The history of popular culture in Chile: different paths”, en Kenneth Aman y Cristian Parker (eds.), Popular culture in Chile. Resistance and survival. Westview Press, Boulder.

Saldivia, Zenobio (2009), "El rol social de las revistas científicas chilenas en el siglo XIX y su contribución a la idea de nación”, en Gabriel Cid y Alejandro San Francisco (eds.), Nación y Nacionalismo en Chile. Siglo $X I X$. Centro de Estudios Bicentenario, Santiago, Vol. 2.

Salgado, Alfonso (2010), "Memoria, heroicidad y nación: monumentos, topónimos, estampillas, monedas y billetes en Chile, 1880-1930”, Bicentenario. Revista de Historia de Chile y América, Vol. 9, º 2.

Salinas, Maximiliano (2004), “Los rotos y la nación: Juan Rafael Allende entre la Guerra del Pacífico y la Guerra civil de 1891”, Mapocho, N 55.

San Francisco, Alejandro (2006), “La apoteosis de Balmaceda. Desde la tumba solitaria a la gloria (Santiago, 1896)”; en Carmen Mc Evoy (ed.), Funerales republicanos en América del Sur: tradición, ritual y nación, 1832-1896. Centro de Estudios Bicentenario, Santiago.

Ídem (2009), “La excepción honrosa de paz y estabilidad, de orden y libertad. La autoimagen política de Chile en el siglo XIX”, en Gabriel Cid y Alejandro San Francisco (eds.), Nación y nacionalismo en Chile. Siglo $X I X$. Centro de Estudios Bicentenario, Santiago, Vol. 1. 
Sanhueza, Carlos (2006), Chilenos en Alemania y alemanes en Chile. Viaje y nación en el siglo XIX. DIBAM/LOM, Santiago.

Sater, William F. (1973), The Heroic Image in Chile: Arturo Prat, Secular Saint. University of California Press, Berkeley.

Schell, Patience (2009), "Museos, exposiciones y la muestra de lo chileno en el siglo XIX”, en Gabriel Cid y Alejandro San Francisco (eds.), Nación y nacionalismo en Chile. Siglo XIX. Centro de Estudios Bicentenario, Santiago, Vol. 1.

Ídem (2001), “Capturing Chile: Santiago’s Museo Nacional during the nineteenth century,” Journal of Latin American Cultural Studies, Vol. 10, $\mathrm{N}^{\circ} 1$.

Serrano, Sol (1994), Universidad y nación. Chile en el siglo XIX. Editorial Universitaria, Santiago.

Silva, Bárbara (2006), "Símbolos y discursos en torno a la nación. Patria Vieja y Centenario”, en Tesis Bicentenario 2004. Colección Tesis Bicentenario, Santiago.

Skuban, William E. (2007), Lines in the sand. Nationalism and identity on the Peruvian-Chilean frontier. University of New Mexico Press, Albuquerque.

Smith, Anthony D. (2004), Nacionalismo: teoría, ideología, historia. Alianza, Madrid.

Smith, Anthony D. (1993), National identity. University of Nevada Press, Reno.

Solberg, Carl. (1970), Immigration and nationalism. Argentina and Chile, 1890-1914. University of Texas Press, Austin.

Sommer, Doris (2004), Ficciones fundacionales. Las novelas nacionales de América Latina. Fondo de Cultura Económica, Bogotá.

Stuven, Ana María (2006), “Guerreros y sabios al panteón republicano: los funerales de José Miguel Infante y Andrés Bello”, en Carmen Mc Evoy (ed.), Funerales republicanos en América del Sur: tradición, ritual y nación, 1832-1896. Centro de Estudios Bicentenario, Santiago.

Ídem (2009), “La cuestión social y la consolidación de la nación: el problema de la inclusión civil y política”, en Ana María Stuven y Marco A. Pamplona (eds.), Estado y nación en Chile y Brasil en el siglo XIX. Ediciones Universidad Católica de Chile, Santiago.

Ídem (1987), “La generación de 1842 y la conciencia nacional chilena”, 
Revista de Ciencia Política, Vol. IX: N 1.

Subercaseaux, Bernardo (1981), "Nacionalismo literario, realismo y novela en Chile (1850-1860)”, Literatura Chilena, Creación y Crítica, N XV.

Tala, Pamela (2001), "La construcción de la identidad nacional en la lira popular: los versos de Rosa Araneda”, Revista Chilena de Literatura, Nº 58.

Torres, Rodrigo (2008), “Zamacueca a toda orquesta. Música popular: espectáculo público y orden republicano en Chile (1820-1860)”, Revista Musical Chilena, N$^{\circ} 209$.

Vergara, Jacinta (2009), “Desde el bastidor al imaginario nacional: Rugendas y la representación de la identidad chilena”, en Gabriel Cid y Alejandro San Francisco (eds.), Nación y nacionalismo en Chile. Siglo XIX. Centro de Estudios Bicentenario, Santiago, Vol. 1.

Vial Correa, Gonzalo (1966), "La formación de las nacionalidades hispanoamericanas como causa de la independencia”, Boletín de la Academia Chilena de la Historia, $\mathrm{N}^{\circ} 75$.

Vicuña, Manuel (2009), Un juez en los infiernos. Benjamín Vicuña Mackenna. Ediciones Universidad Diego Portales, Santiago.

Voionmaa, Liisa Flora (2004), Escultura pública: del monumento conmemorativo a la escultura urbana, Santiago 1792-2004. Ocho Libros Editores, Santiago.

Woll, Allen (1982), A functional past: the uses of history in nineteenthcentury Chile. Louisiana State University Press, Baton Rouge.

Wood, James A. (2009), “Guardias de la nación: nacionalismo popular, prensa política y la guardia cívica de Santiago, 1826-1846”, en Gabriel Cid y Alejandro San Francisco (eds.), Nación y nacionalismo en Chile. Siglo XIX. Centro de Estudios Bicentenario, Santiago, Vol. 2.

Yaeger, Gertrude (1981), Barros Arana's Historia jeneral de Chile: Politics, history, and national identity. Texas Christian University Press, Fort Worth.

Zaldívar, Trinidad y Sánchez, Macarena (2009), “Símbolos, emblemas y ritos en la construcción de la nación. La fiesta cívica republicana: Chile 1810-1830”, Gabriel Cid y Alejandro San Francisco (eds.), Nación y nacionalismo en Chile. Siglo XIX. Centro de Estudios Bicentenario, Santiago, Vol. 2.

Recibido: 22.12.2011

Aceptado: 21.03.2012 